\title{
IDENTIFICACIÓN DE ISLAS DE CALOR EN LA CIUDAD DE LIMA METROPOLITANA UTILIZANDO IMÁGENES DEL SATÉLITE LANDSAT $5 \mathrm{TM}$
}

\author{
IDENTIFYING OF HEAT ISLANDS IN THE CITY OF METROPOLITAN LIMA USING \\ SATELLITE IMAGES 5TM
}

${ }^{1}$ Vanessa Sofía Soberón Forsberg y ${ }^{2}$ Esaúl Obregón Párraga

\begin{abstract}
Resumen
Lima Metropolitana cuenta con muy pocas estaciones meteorológicas, lo cual dificulta obtener temperaturas de la superficie en forma distribuida e imposibilita la identificación de las Islas de Calor (IC). Sin embargo, mediante el uso de imágenes del satélite LANDSAT 5TM, pudimos obtener la temperatura de superficie e identificar las IC, analizando para ello seis eventos que fueron corregidos por emisividad.

Los eventos analizados corresponden a los registrados a las 10:00 a.m. en las siguientes fechas: 11 de febrero del 2008, 15 de abril del 2008, 1 de mayo del 2008, 2 de abril del 2009, 31 de enero del 2010 y 7 de marzo del 2011. Asimismo, se obtuvieron datos de temperatura del aire de 6 estaciones meteorológicas para estos eventos.

Se encontró islas de calor negativas durante la mañana principalmente en las zonas no urbanas. Se ubicaron 5 islas de calor urbanas, 7 islas en zonas industriales y comerciales, y 3 micro islas de calor urbanas.

La asociación entre temperatura del aire, temperatura de superficie y el Índice de Vegetación de Diferencia Normalizada (NDVI) fue muy baja, mientras que el coeficiente de correlación mejora para la relación del NDVI y la diferencia entre la temperatura de superficie con la temperatura del aire, obteniéndose coeficientes de entre $0.13(01 / 05 / 2008)$ y hasta $0.89(15 / 04 / 2008)$.
\end{abstract}

Palabras clave: Islas de calor urbana, Landsat 5TM, Lima Metropolitana y emisividad.

\begin{abstract}
The fact that Metropolitan Lima has very few weather stations makes it extremely difficult to obtain surface temperatures in a distributed manner and prevents the identification of Heat Islands (HI). However, using LANDSAT 5TM satellite images, we were able to obtain the surface temperature of various areas and to identify HI, analyzing six events which were corrected for emissivity.

The events analyzed were those recorded at 10:00 a.m. on the following dates : February 11, 2008; April 15, 2008; May 1, 2008; April 2, 2009; January 31, 2010 and March 7, 2011. Moreover, air temperature data from 6 meteorological stations were also obtained for these events.

Negative heat islands were found in Metropolitan Lima in the morning, mainly in non-urban areas. On the other hand, five heat urban islands, seven islands in industrial and commercial areas, and 3 urban micro heat islands were identified.

The association between air temperature, surface temperature, and the Normalized Difference Vegetation Index (NDVI) was very low, whereas the correlation coefficient between the NDVI and the difference between the surface temperature and the brightness temperature improved, with coefficients between 0.13 (May 1, 2008) and 0.89 (April 15, 2008) being observed.
\end{abstract}

Keys words: Urban Heat Island, Landsat 5TM, Metropolitan Lima and emissivity. 


\section{Introducción}

En la última década el aumento poblacional en las ciudades ha generado una alta demanda de viviendas, para satisfacer, las áreas verdes (jardines y parques) son reemplazadas por condominios y edificaciones multifamiliares. Este cambio debido al sector construcción afecta al clima urbano produciendo un nuevo fenómeno, conocido como islas de calor urbanas; las ciudades almacenan calor causando un aumento de temperaturas que afecta la salud de las personas, la emisión de gases de efecto invernadero y el consumo de energía (Galindo et al; 2010). Las causas principales han sido la morfología de la ciudad, la contaminación del aire y los parámetros meteorológicos. También existe el fenómeno inverso conocido como isla de calor negativa, en donde el entorno no urbano tiene una temperatura más elevada que el área urbana (Pérez y Peña, 2010).

La importancia de estudiar las islas de calor urbanas radica en aportar conocimientos que pueden contribuir a mejorar el confort térmico en las ciudades para que sus habitantes puedan tener un ambiente más agradable. Es importante conocer la presencia de las islas de calor porque estas pueden afectar y perjudicar a la salud de las personas (EPA, 2007). El aumento de temperatura genera un mayor consumo de energía por la utilización de refrigerantes y en consecuencia hay un mayor gasto económico y se liberaran gases de efecto invernadero (EPA, 2007). El aumento de temperatura también acelera la velocidad de las reacciones químicas de los procesos de combustión produciendo gases contaminantes que pueden ser dañinos para el hombre, como el ozono O3 (Streutker, 2003). Todos estos factores y procesos ambientales son importantes para el ordenamiento territorial.

El presente trabajo de investigación tiene como objetivo, identificar islas de calor urbanas superficiales en la ciudad de Lima Metropolitana y la relación entre la temperatura de superficie y la temperatura del aíre.

La temperatura de superficie se obtuvo a partir de los radiancia de los datos del satélite Landsat 5TM, pasando a la temperatura de brillo y corregida por emisividad para los días: 7 de marzo del 2011, 31 de enero del 2010, 2 de abril del 2009, 11 de febrero del 2008, 1 de mayo del 2008 y 15 de abril del 2008.

\section{Materiales y métodos}

\section{Área de estudio y las Islas de Calor.}

Lima es la capital del Perú y está situada en la región occidental de América del sur a orillas del Océano Pacífico. Lima metropolitana tiene una extensión aproximada de $2819 \mathrm{~km}^{2}$, se encuentra constituida por los distritos de la provincia de Lima y de la Provincia Constitucional del Callao.
Según el Atlas Ambiental de Lima (2007), el clima de Lima es húmedo con poca lluvia y tiene temperaturas moderadas ocasionadas por el afloramiento de aguas frías de la Corriente Peruana. Los factores naturales que influyen en la ciudad de Lima metropolitana son la Cordillera de los Andes, la capa de inversión térmica, la topografía, el efecto de continentalidad y la corriente de Humboldt. Las variables climáticas que influyen en el clima son la temperatura, la precipitación, radiación solar, humedad y viento.

Barros (2010) realizó un estudio para identificar la presencia de las islas de calor urbanas atmosféricas para dos días típicos de verano y encontró dos núcleos cálidos con la temperatura del aire a las 16:00 horas, ubicados entre los distritos de Independencia y Rímac y entre los distritos de Ate Vitarte, Santa Anita, El Agustino y San Luis. También encontró sólo un núcleo térmico para las 19:00 horas, entre los distritos de la Independencia, Rímac, San Martín de Porres y Los Olivos.

El área específica de estudio se delimitó de tal manera que se cuente con zona urbana y zona rural con presencia de suelos de uso comercial e industrial y suelo desnudo; esto corresponde a la ciudad de Lima Metropolitana; tal como se muestra en la Figura 1.

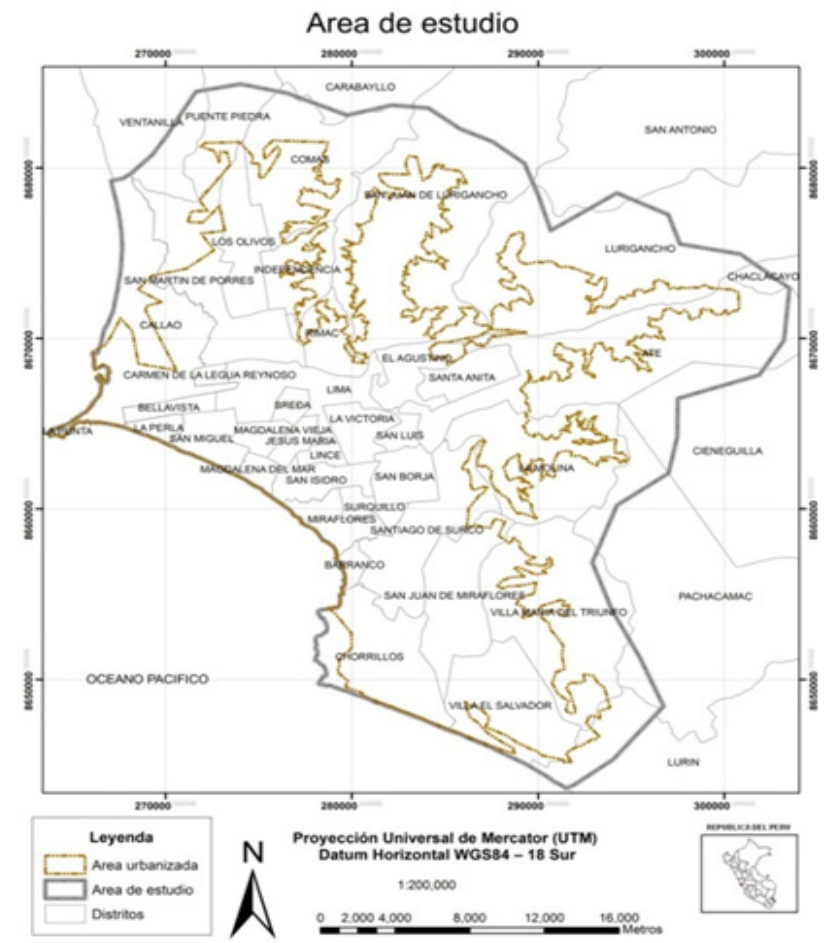

Figura 1. Delimitación del área de estudio.

\section{Datos de las imágenes del Satélite y procesamiento.}

La temperatura se calculó a partir de los datos de la banda 6 (termal; 10.4-12.5 $\mu \mathrm{m}$ ) del satélite Landsat 5 TM proveniente de United States Geological Survey 
(USGS), cuya información se tenía acceso libre a la data y presenta una resolución espacial de 120 metros, también está disponible los datos transformados a pixeles de $30 \times 30 \mathrm{~m}$. Estos datos provenientes de dicho satélite, fueron seleccionados las fechas a la 15:00 horas GTM (hora local de 10:00 am. en Lima Perú): 11 de febrero del 2008; 15 de abril del 2008; 01 de mayo del 2008; 02 de abril del 2009; 31 de enero del 2010 y 7 de marzo del 2011. Asimismo se obtuvo datos de las bandas 3 y 4 del mismo satélite para las fechas seleccionadas.

Para el pre procesamiento de los datos.- los eventos elegidos corresponden a días sin presencia de nubes o mínimas, por ello no fue necesario realizar correcciones atmosféricas; asimismo se utilizó las imágenes con corrección geométrica hasta el nivel L1T, se verificó el cumplimiento del protocolo de imágenes Landsat, según CDC-UNALM (2011) y el protocolo del Ministerio del Ambiente (2014) donde mencionan que los productos de los datos L1T consisten en productos de datos L1R con correcciones geométricas sistemáticas aplicadas, utilizando para ello puntos de control terrestre (GCP) o información de posición integrada a una proyección cartográfica referenciada a WGS84, adicionalmente los datos también contienen una corrección topográfica por desplazamiento del terreno debido al relieve, concluyendo que el resultado final es un producto geométricamente rectificado libre de distorsiones relacionados con el sensor, solo será necesario corregir en XY de ser necesario, debido al tipo de variable (temperatura) y al contrastar con la información con otras cartografías con el programa ArcGis Desktop 9.3 se procedió a proyectar las imágenes al sistema WGS 1984 UTM 18 Sur.

Según Fernandes G. y Moreira de Souza N. (2013 mencionan que Cunha et al. empleó la ecuación 1 para calcular la radiancia espectral (Lsen) para las bandas 1, 2, 3, 4, 5, 6 y 7 del TM Landsat 5.

$$
\text { Lsen }=\mathrm{Lmin}+((\operatorname{Lmax}-\mathrm{Lmin}) / 255) \mathrm{ND}
$$

Para el presente trabajo se utilizó la banda 6, las variables y unidades son:

Las unidades de Lsen es, $\mathrm{W} /\left(\mathrm{m}^{2} \mathrm{sr}^{1} \mu \mathrm{m}^{1)}\right.$; $\mathrm{ND}$ es el número digital de la banda 6 relacionado a la intensidad del pixel, variando de 0 a 255; Lmax y Lmin son las radiancias espectrales máximas y mínimas presentadas por Chander et al. (2009) cuyos valores que corresponden para la banda 6 son $\operatorname{Lmax}=15.303$ y $\operatorname{Lmin}=1.2378$.

La radiancia espectral de la ecuación 1 , se convierte a temperatura de brillo $(\mathrm{Tb})$ o temperatura del satélite, en grados Kelvin $\left({ }^{\circ} \mathrm{K}\right)$, según Sospedra et al. (1998) citado por Martinez (2010); la temperatura de brillo para la banda 6 del satélite Landsat se calcula con la ecuación 2.

$$
\mathrm{Tb}=\mathrm{k}_{2} / \ln \left(\mathrm{k}_{1} / \text { Lsen }+1\right)
$$

Dónde: $K 1$ y $K 2$ son constantes de calibración; Chander et al. (2009) presenta para el satélite Landsat 5 TM los valores para $K 1=607.76 \mathrm{~W} /\left(\mathrm{m}^{2} \mathrm{sr}^{1} \mu \mathrm{m}^{1}\right)$ y $\mathrm{K}_{2}=1260.56$ ${ }^{\circ} \mathrm{K}$, Lsen, la radiancia calculada en la ecuación 1 y $\ln$ es el logaritmo natural.

\section{Cálculo de la temperatura de superficie (TST)}

La TST es un factor importante para una gran variedad de aplicaciones como: hidrología, agricultura, bioquímica, cambios climáticos, etc. Para su medición por medio de imágenes satelitales se requiere de cierto procedimiento y condiciones; para el presente trabajo no se realizó la corrección atmosférica debido a que la presencia de nubes no fue significativa en la zona de estudio los días seleccionados. Se realizó la corrección por emisividad utilizando el método de Stathopoulou et al., citado por Farina A. (2012); este método considera el NDVI.

El Índice de Vegetación de la Diferencia Normalizada (NDVI), se utiliza principalmente en dos situaciones, i) para disminuir el efecto de relieve (topográfico) que no es nuestro caso y el ii) para mejorar la discriminación entre dos cubiertas del suelo con diferente comportamientos espectral en dos bandas, de esta manera realzar suelos y vegetación en el visible e infrarrojo cercano del espectro electromagnético, por ello se produce un notable contraste espectral entre la baja reflectividad de la banda roja del espectro y la banda del infrarrojo cercano, lo que permite separar con relativa claridad la vegetación de otras cubiertas o suelos desnudos. El método más empleado para el cálculo del NDVI es el que presenta Chuvieco (2000) según la siguiente relación:

$$
\mathrm{NDVI}=(\mathrm{IRC}-\mathrm{R}) /(\mathrm{IRC}+\mathrm{R})
$$

Siendo IRC, la reflectividad en la banda infra rojo cercano y $R$, la reflectividad en la banda rojo.

Según Guyot y Gu. citado por Chuvieco (2000), mencionan que los valores de NDVI para imágenes Lansat calculados a partir de ND subestiman entre 0.05 y 0.20 el valor calculado con reflectividades, siendo este error mayor para valores de NDVI inferiores a 0.5 , por lo que propone la siguiente corrección para imágenes de Lansat-TM.

$$
\mathrm{NDVI}=\left(\mathrm{ND}_{\mathrm{IRC}}-0.801 \mathrm{ND}_{\mathrm{R}}\right) /\left(\mathrm{ND}_{\mathrm{IRC}}+0.801 \mathrm{ND}_{\mathrm{R}}\right)
$$

El NDVI de la ecuación 4, está corregido, y los valores de NDIRC y NDR corresponden a los valores digitales de las bandas 4 y 3 respectivamente para el satélite Landsat 5 TM.

La corrección de emisividad según el método de Stathopoulou et al., (2007) considera tres escenarios de acuerdo a lo indicado en el cuadro 1 . 
Cuadro 1. Resumen del cálculo de emisividad ( $(\varepsilon)$.

\begin{tabular}{|c|c|c|}
\hline NDVI & PIXELES & EMISIVIDAD \\
\hline NDVI $<0.2$ & $\begin{array}{l}\text { Si el NDVI tiene valores menores a } 0.2 \text {, los pixeles son considerados como } \\
\text { suelo urbano. }\end{array}$ & 0.92 \\
\hline $0.2 \leq \mathrm{NDVI} \leq 0.5$ & $\begin{array}{l}\text { Cuando el NDVI tiene valores mayores o igual a } 0.2 \text { y menor o igual a } 0.5 \text {, } \\
\text { los pixeles son considerados mixtos entre vegetación y suelo urbano y su } \\
\text { emisividad }(\varepsilon) \text { se calcula según las ecuaciones: }\end{array}$ & $\begin{array}{c}\text { Ecuación } 5 \\
\varepsilon \mathrm{v}=0.98 \\
\varepsilon \mathrm{m}=0.92 \\
\text { Pv, Ecuación } 6 \\
\mathrm{~d} \varepsilon \text {, Ecuación } 7\end{array}$ \\
\hline $\mathrm{NDVI}>0.5$ & $\begin{array}{l}\text { Cuando el NDVI tiene valores mayores a } 0.5, \text { los pixeles son considerados } \\
\text { de vegetación. }\end{array}$ & 0.98 \\
\hline
\end{tabular}

La emisividad $(\varepsilon)$ es calculada en base a la siguiente ecuación:

$\varepsilon=\varepsilon v P v+\varepsilon m(1-P v)+d \varepsilon$

Donde $\varepsilon v$ es la emisividad de la vegetación y su valor típico es 0.98; $\mathrm{em}$ es la emisividad del suelo urbano su valor típico se asume $0.92 ; \mathrm{Pv}$ es la proporción de la vegetación y de es la fracción de emisividad, estos dos últimos se obtienen de las ecuaciones 6 y 7.

$\mathrm{PV}=(\mathrm{NDVI}-0.2)^{2} /(0.5-0.2)^{2}$

$\mathrm{d} \varepsilon=(1-\varepsilon \mathrm{m}) \varepsilon \mathrm{v} 0.55(1-\mathrm{Pv})$

Artis y Carnahan citado por Farina (2012) encontraron una fórmula (ecuación 8) para calcular la temperatura de superficie (TST) en ${ }^{\circ} \mathrm{K}$, en función de la temperatura de brillo (Tb) corregida por emisividad; para ello se utilizó la información de la banda 6, con píxeles de 30x30 m, obtenida de la misma fuente mencionada anteriormente.

$\mathrm{TST}=\mathrm{Tb} /(1+(\lambda \mathrm{Tb} / \rho) \ln \varepsilon)$

Siendo, $\lambda$ es la longitud de onda de la radiancia emitida $(11.5 \mu \mathrm{m}), \varepsilon$ es la emisividad y $\rho$ tiene un valor de $1.438 \times 10^{-2} \mathrm{~m}^{* 0} \mathrm{~K}$ que se obtuvo mediante la ecuación 9 .

$\rho=\mathrm{ch} / \sigma$

Donde, h es la constante de Planck $\left(6.26 \times 10^{-34} \mathrm{~J} \mathrm{~s}\right)$, c es la velocidad de la luz $\left(2.998 \times 10^{8} \mathrm{~m} / \mathrm{s}\right), \sigma$ es la constante de Stefan Boltzmann $\left(1.38 \times 10^{-23} \mathrm{~J}^{\mathrm{o}} \mathrm{K}^{-1}\right)$.

La temperatura de brillo y de superficie, en grados Celsius, se calcula restando 273.15 a la temperatura de la ecuación 2 y ecuación 8 , respectivamente.

Elaboración de mapas térmicos y la determinación de la relación entre la temperatura de superficie, la temperatura del aire y el NDVI.

Con el software ArcGis Desktop 9.3 se elaboró seis mapas de temperatura de brillo a partir de las imágenes Landsat, con dicha información se encontraron mapas de temperatura de brillo y de superficie en base a sus correspondientes datos; a partir de esta información se ubicaron las islas de calor superficiales en la ciudad de Lima Metropolitana.

Para analizar las islas, en forma conjunta, se elaboró un mapa representativo basado en los seis eventos, este mapa nos permite ubicar con mayor precisión tanto las islas de calor. Se consideró como islas de calor urbanas a aquellas superficies que tienen una temperatura mayor al promedio de la temperatura encontrada en el área urbanizada.

Los datos de temperatura del aire, fueron proporcionados por el Servicio Nacional de Meteorología e Hidrología (Senamhi) y de la Estación Meteorológica Alexander Von Humboldt (OVH). En la Tabla 1 se presenta la ubicación geográfica de estas estaciones meteorológicas.

Para realizar la correlación entre los datos de temperatura del aíre y temperatura de superficie, es necesario que los datos se encuentren en el mismo sistema de coordenadas; con las herramientas del ArcGis Desktop 9.3 se puso el sistema de coordenadas de las estaciones en el mismo sistema de coordenadas de las imágenes Landsat 5 TM (UTM 18 Sur, WGS 84).

Para determinar los estadísticos, como el coeficiente de correlación y determinación y otros se usó el software "Statistical Package for Social Sciences" (SPSS) se elaboraron gráficos de dispersión entre las variables: temperatura del aire; temperatura de brillo; temperatura de superficie; y del NDVI.

Se elaboró mapas de temperatura de superficie (TST) en ${ }^{\circ} \mathrm{C}$, para cada evento de acuerdo a la metodología descrita anteriormente, este resultado se muestra en la Fig. 3.

\section{Coeficiente de Determinación $\left(\mathbf{r}^{2}\right)$}

Es lo que explica un modelo de la realidad, varía de 0 a 1 ; será 1 cuando el modelo es perfecto y explica el 100\%. Es decir cuánto se explica una variable dependiente por una o más variables independientes. Para una variable independiente el cálculo de r2, está dado por la ecuación 10. 
Tabla 1: Ubicación geográfica de las estaciones meteorológicas.

\begin{tabular}{lccc}
\hline \multicolumn{1}{c}{ Estación Meteorológica } & Latitud Sur & Longitud Oeste & Altura (m.s.n.m.* \\
\hline Las Palmas & $12^{\circ} 09^{\prime}$ & $77^{\circ} 01^{\prime}$ & 73 \\
Campo de Marte & $12^{\circ} 04^{\prime}$ & $77^{\circ} 02^{\prime}$ & 123 \\
Hospital Central de la FAP & $12^{\circ} 06^{\prime}$ & $77^{\circ} 01^{\prime}$ & 158 \\
Alexander Von Humboldt & $12^{\circ} 05^{\prime}$ & $76^{\circ} 56^{\prime}$ & 257.43 \\
Lima-Este & $12^{\circ} 03^{\prime}$ & $77^{\circ} 55^{\prime}$ & 241 \\
Collique & $11.931392^{\circ}$ & $77.064720^{\circ}$ & 138 \\
\hline
\end{tabular}

* m.s.n.m. $=$ metros sobre el nivel del mar

$$
r^{2}=\frac{\sum_{i=1}^{n}(x-\bar{x})(y-\bar{y})}{\sqrt{\sum_{i=1}^{n}(x-\bar{x})^{2}(y-\bar{y})^{2}}}
$$

Siendo, y la variable dependiente con media $\bar{y}$; x la variable independiente con media $\bar{x}$ y n el número de pares de observaciones o eventos.

A r se le denomina coeficiente de correlación donde mide el grado de asociación lineal entre las dos variables, $x-y$ $(-1 \leq \mathrm{r} \leq 1)$.

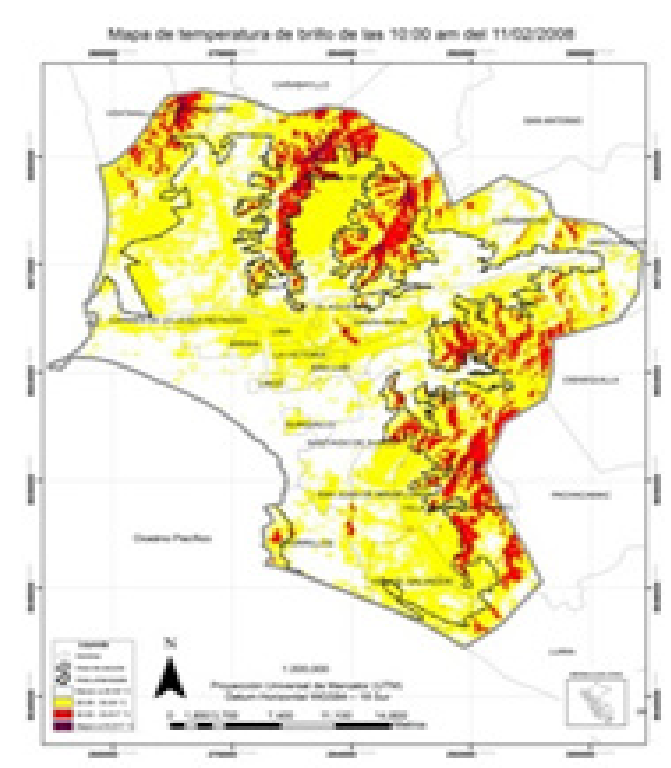

\section{Resultados y discusión}

Determinación de la distribución espacial de las islas de calor

En base a la información satelital y al método planteado se obtuvo la temperatura de brillo $\left(\mathrm{en}^{\circ} \mathrm{C}\right)$ para cada evento, tal como se muestra en la figura 2.

Como se puede observar los indicios de la presencia de núcleos de bajas temperaturas, temperaturas medias y otros núcleos de altas temperaturas dentro de Lima Metropolitana pero en forma distribuida espacialmente en los eventos utilizados. Asimismo se observa que los núcleos que presentan coinciden espacialmente, unos con mayor influencia que otros.

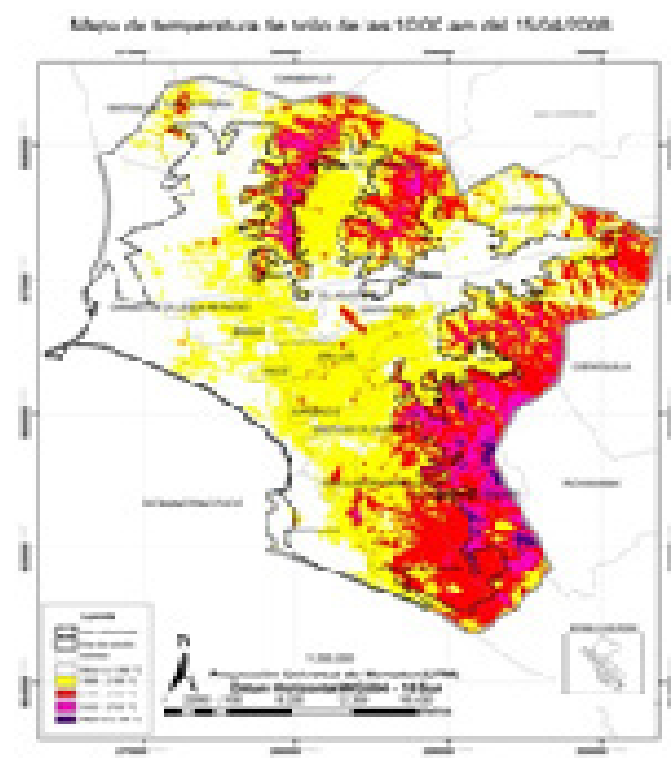

Figura 2. Temperatura de brillo para lima metropolitana con datos de satélite LANDSAT 5 TM. 
IDENTIFICACIÓN DE ISLAS DE CALOR EN LA CIUDAD DE LIMA METROPOLITANA UTILIZANDO IMÁGENES DEL SATÉLITE LANDSAT 5TM

Continuación de la Figura 2
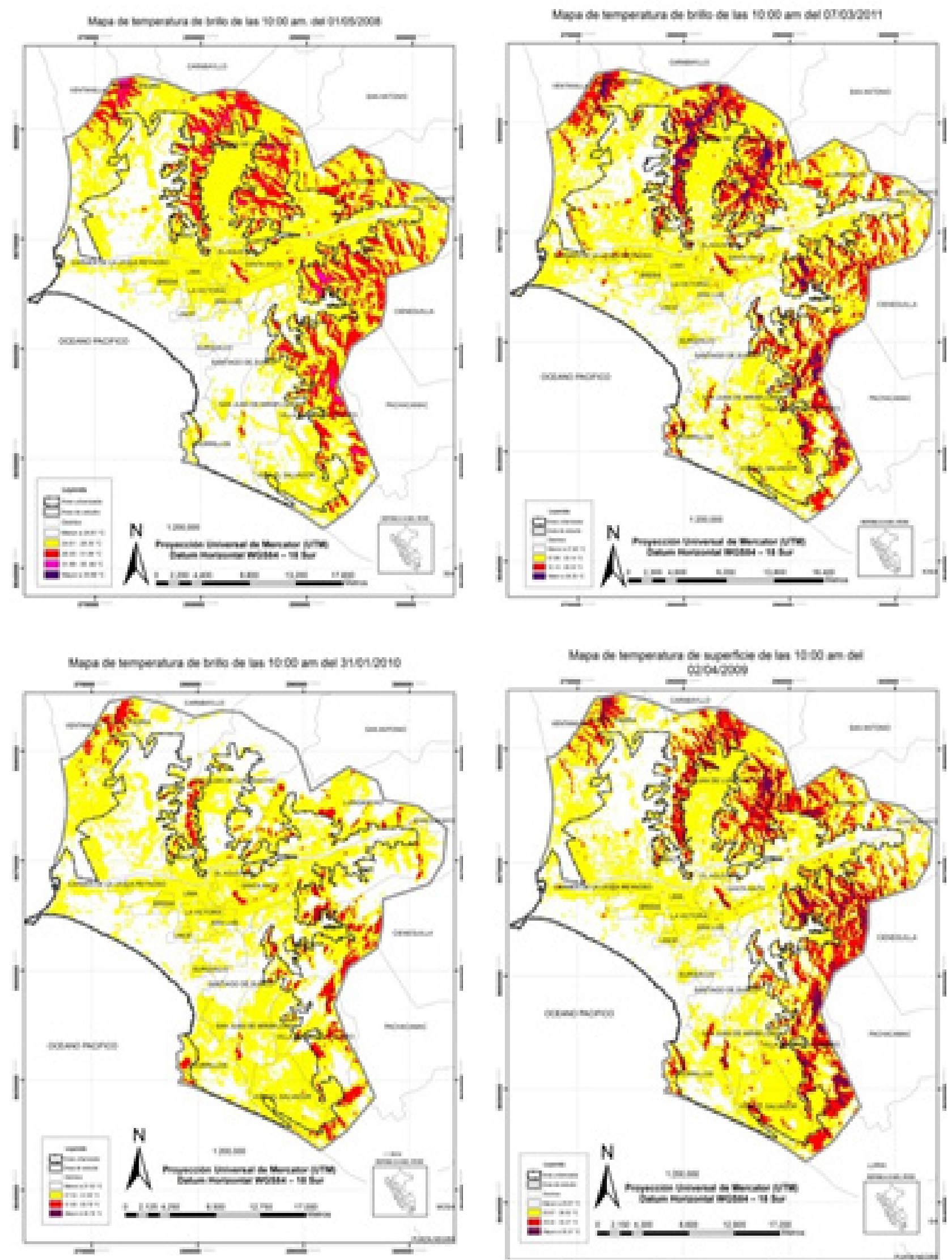

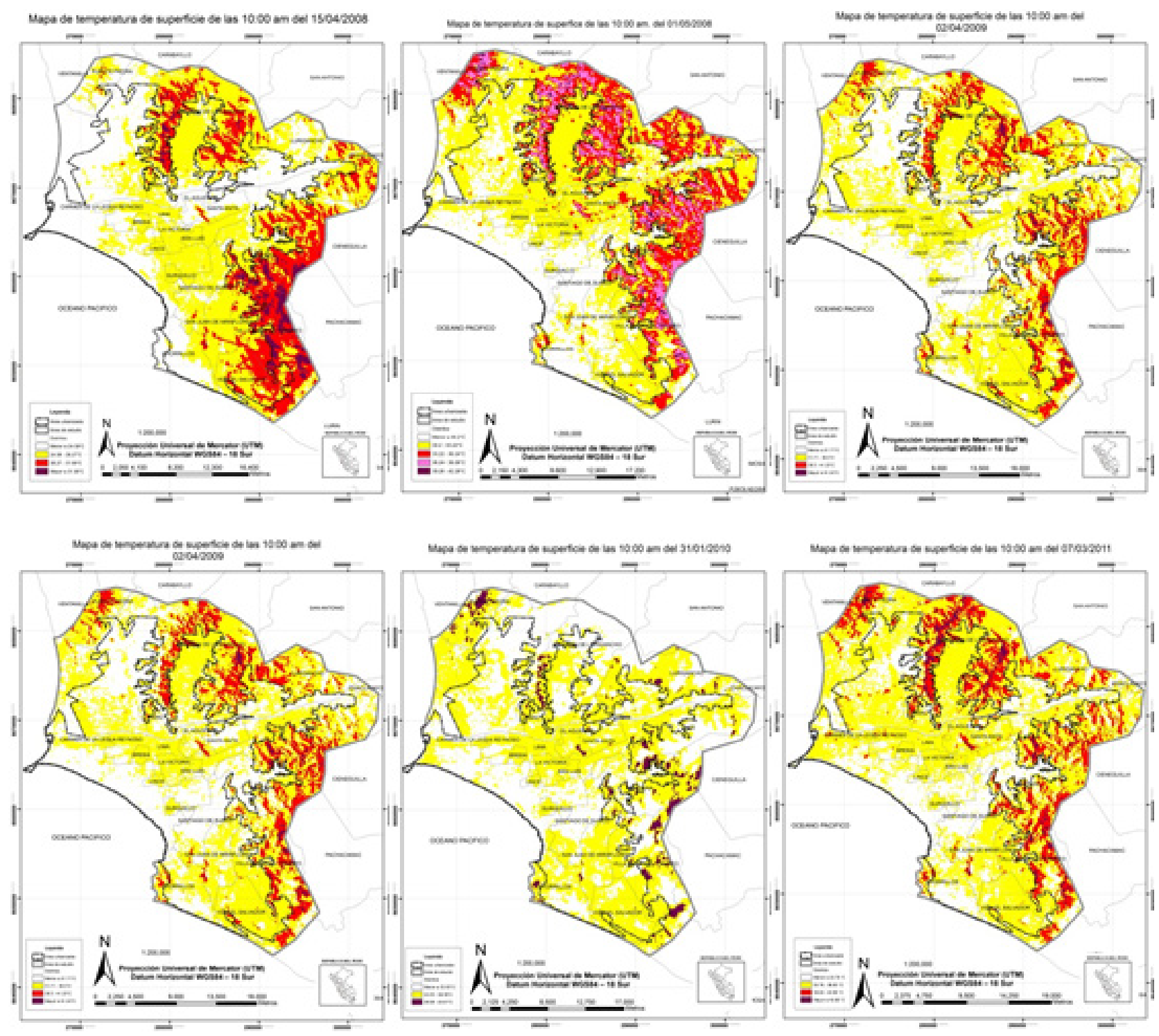

Figura 3. Temperatura De Superficie, En ${ }^{\circ} \mathrm{C}$, Para Lima Metropolitana.

Se observa, en la figura 3, que la temperatura de superficie en todos los casos calculados es menor cerca del mar y a medida que nos vamos alejando de él hacia el este, la temperatura aumenta, hay concordancia con lo mencionado por Cisneros y Quezada citado por Quezada (1987) que esto es debido al efecto de continentalidad al igual encontró Barros (2010); y la misma tendencia encontró Quezada (1987) que las isotermas de temperatura del aire tienen menor valor conforme se acerca al océano Pacífico.

Las temperaturas de superficie obtenidas son muy alta, en algunos casos superan los $40^{\circ} \mathrm{C}$, estos valores serán referenciales, debido a que el objetivo del trabajo es la localización de las islas de calor, sin embargo esta altas temperaturas son percibidas por las personas y expresadas como sensación térmica. En todos los casos esta temperatura es considerada alta desde el punto de vista de confort, esto indica un elevado calentamiento de la superficie, posiblemente por una elevada densidad poblacional y elevado número de industrias en la ciudad.

Desde el punto de vista de urbanización se encontró temperaturas más elevadas en las zonas no urbanizadas y temperaturas menores en las zonas urbanizadas; lo que indica la presencia de la isla de calor negativa en coincidencia lo mencionado por Pulache y Menis (2009). Estas islas de calor negativas son producidas por la presencia de cerros desnudos, superficies de color oscuro, con propiedades térmicas y caloríficas que durante el día se calientan más que las zonas urbanas. También este comportamiento está dentro lo mencionado por Pérez y Peña (2010) que indican que este efecto corresponde a climas temperados, tropicales, semiáridos y áridos, y ocurren principalmente durante la mañana como es el caso del área en estudio a las 10:00 hrs. 
Se elaboró un mapa representativo basado en los seis eventos tal como se muestra en la Fig. 4.

De las Figura 3 y 4 se observa, los núcleos térmicos con temperaturas más elevadas se encontraron en el cerro El Agustino, en el cerro Gramadal ubicado entre Chorrillos y San Juan de Miraflores y en el distrito de Chorrillos. Sin embargo, estos no son islas de calor urbanas superficiales ya que no están ubicados en zonas urbanas. Las islas de calor con mayor núcleo térmico se encontraron en el extremo noreste de la ciudad, teniéndose el mayor núcleo térmico en el distrito de Ate y LuriganchoChosica, seguido por los distritos de Villa María del Triunfo, Villa el Salvador y San Juan de Miraflores y el distrito de San Juan de Lurigancho. Las coordenadas estos núcleos térmicos se presentan en el tabla 2. No fue posible discutir la relación de temperatura de superficie con las temperaturas de las estaciones meteorológicas, debido a que en los núcleos térmicos no hay información medida proveniente de dichas estaciones y cualquier interpolación puede afectar positiva o negativamente los resultados.

\section{Mapa de temperatura de superficie promedio de las 10:00 am}

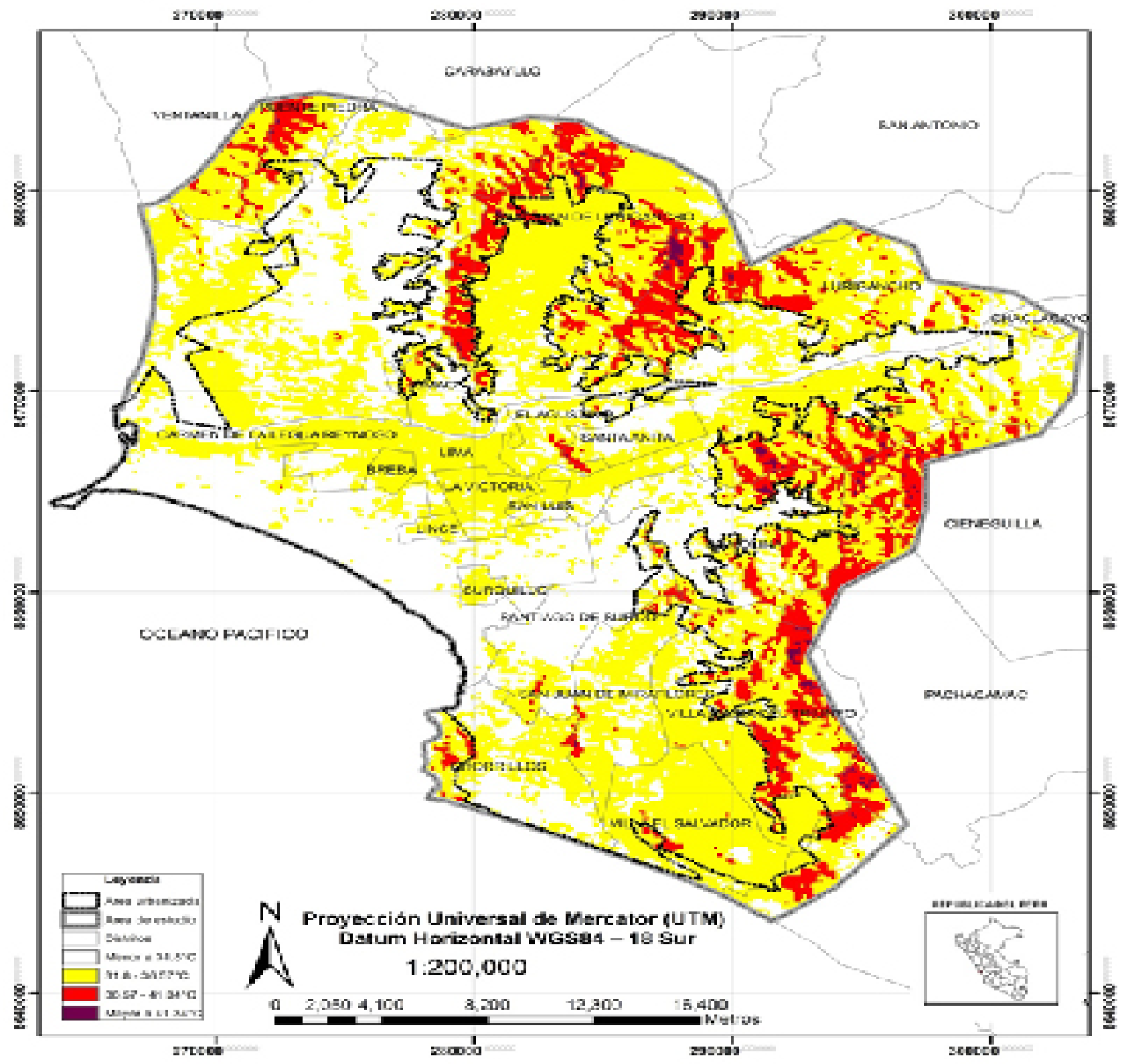

Figura 4. Mapa De Temperatura Promedio De La Superficie $\left({ }^{\circ} \mathrm{C}\right)$ Para Lima Metropolitana A Las 10:00 am. 
Tabla 2. Coordenadas de los núcleos térmicos más elevados.

\begin{tabular}{lccc}
\hline \multirow{2}{*}{ Ubicación (distrito) } & $\begin{array}{c}\text { Temperatura de Superficie } \\
\left({ }^{\circ} \mathrm{C}\right)\end{array}$ & \multicolumn{2}{c}{$\begin{array}{c}\text { Sistema de coordenadas, WGS 84 - UTM 18 Sur } \\
\text { (metros) }\end{array}$} \\
\cline { 3 - 4 } & 36,41 & 291138,45 & Y \\
\hline Ate y Lurigancho-Chosica & 35,90 & 294002,61 & 8667654,57 \\
Ate y Lurigancho-Chosica & 36,34 & 290501,97 & 8669086,65 \\
Villa María del Triunfo & 36,15 & 291774,93 & 8656993,53 \\
Villa María del Triunfo & 36,12 & 281750,37 & 8649037,53 \\
San Juan de Lurigancho & 36,04 & 281591,25 & 8679111,21 \\
San Juan de Lurigancho & 35,91 & 281909,49 & 8679270,33 \\
San Juan de Lurigancho & & & 8678952,09 \\
\hline
\end{tabular}

De los resultados obtenidos, en total se identificó la presencia de 15 islas de calor urbana como son mostrados en la figura 3, estas islas son descritas a continuación:

- Una isla de calor se ubicó en el norte de Lima, en el distrito de San Juan de Lurigancho.

- En el este de Lima se ubicaron dos islas de calor; uno entre los distritos de Ate y Lurigancho-Chosica y otro en el distrito de La Molina.

- En el sur, se ubicó una isla de calor, entre los distritos de San Juan de Miraflores; Villa El Salvador y Villa María del Triunfo.

- En el centro de la ciudad, se encontró una isla de calor entre los distritos de Rímac y El Agustino.

- En las zonas comerciales e industriales de Lima Metropolitana se encontraron siete islas de calor se ubicaron; cuatro islas en la zona industrial y comercial de los distritos del Callao, Carmen de La legua Reynoso, Lima y en Breña; una isla de calor se ubicó en la zona comercial e industrial de La victoria; otra isla de calor se encontró en la zona industrial entre los distritos de San Luis, Ate, El Agustino y Santa Anita; y en el sur se ubicó una isla de calor en la zona industrial del distrito de Surquillo. Esta distribución es concordante con lo indicado por Sarricole (2010) quién afirmó que durante el día las Isla de calor urbana superficial tiene dos núcleos: en el centro de la ciudad y las zonas industriales.

- Se presentaron tres micro islas de calor; ubicadas en el distrito de Santiago de Surco, San Juan de Miraflores y en el Callao (aeropuerto Jorge Chávez); estos lugares se caracterizan por su poca vegetación, techos con baja reflectividad y pistas asfaltadas.

\section{Determinación del coeficiente de correlación (r).}

\section{a) Entre la temperatura del aire $\left({ }^{\circ} \mathrm{C}\right)$ y la temperatura de superficie $\left({ }^{\circ} \mathrm{C}\right)$}

Para determinar algún estadístico, se tiene muy poca información, en Lima metropolitana de los datos de temperatura del aire, debido al bajo número de estaciones. Una de las alternativas es interpolar los datos y el trazado de isotermas para contrastar con la imagen de las temperaturas de superficie encontradas; sin embargo esto puede generar grandes errores debido a la alta variabilidad espacial de la temperatura superficial respecto a la del aire; con esta baja disponibilidad de información se encontró los coeficientes de correlación para los eventos estimados, tal como se muestra en la tabla 3 y con coeficiente de asociación menor a 0.6 y según la definición de coeficiente de determinación, que menos del $40 \%$ de la variabilidad de la temperatura de la superficie depende de la variabilidad de la temperatura del aire.

Este resultado de las bajas correlaciones coincide con los mencionados por: Roth et al. (1989) y Goldreich (1985), donde indican que no hay una conexión simple entre ambas variables, y con lo mencionado por Li et al. (2008) quiénes mencionan que no se puede utilizar la temperatura de superficie como sustituto directo de la temperatura del aire debido a las altas diferencias de los valores absolutos entre estas dos variables.

La temperatura de superficie supera a la temperatura del aire, en los casos analizados, a excepción del día 15 de abril de 2008, sin embargo no existe ningún comportamiento similar definido por estas variables o que muestra una baja correlación entre ambas variables.

Tabla 3. Coeficiente de correlación (r) y de Determinación (r2) en las relaciones entre la temperatura de superficie $\left({ }^{\circ} \mathrm{C}\right)$ (Var. dependiente) y la temperatura del aire $\left({ }^{\circ} \mathrm{C}\right)$ (Var. independiente).

\begin{tabular}{ccc}
\hline Fecha & $\mathrm{r}$ & $\mathrm{r}^{2}$ \\
\hline $11 / 02 / 2008$ & 0,155 & 0,024 \\
$15 / 04 / 2008$ & 0,435 & 0,189 \\
$01 / 05 / 2008$ & 0,467 & 0,218 \\
$31 / 01 / 2010$ & 0,566 & 0,321 \\
$07 / 03 / 2011$ & 0,581 & 0,338 \\
\hline
\end{tabular}

Como se pudo observar que el coeficiente de determinación (r2) es bajo, entre la temperatura de 
superficie y la temperatura del aire, asimismo se obtiene una correlación baja debido al comportamientos distintos de cada variable al mismo tiempo no es posible realizar mayor análisis ni conclusiones debido al bajo número de datos y a otros factores no analizados en esta investigación como por ejemplo: tipos de vegetación, material de superficie, albedo, etc. que influyen en el resultado.

\section{b) Entre el NDVI con la temperatura de superficie $\left({ }^{\circ} \mathrm{C}\right)$ y la temperatura del aire $\left({ }^{\circ} \mathrm{C}\right)$}

La relación entre la temperatura de superficie y el NDVI fue muy baja, donde el coeficiente de correlación apenas llegó a 0.5 .

Para Sun y Kafatos (2007); mencionan que en teoría se espera una correlación alta; negativa para los meses de verano y positiva para invierno sin embargo para Barros (2010) las bajas correlaciones para Lima Metropolitana se debe a la poca presencia de áreas verdes.

Se relacionó la temperatura de superficie con las otras variables generando un gran número de posibilidades entre ellos. Se encontró una mejora relativa de los coeficiente se correlación entre la diferencia entre la Temperatura de superficie con la temperatura del aire versus el NDVI.

El resultado del cálculo de los coeficientes de correlación por eventos, tal como se muestra en el tabla 4; varían entre 0.13 y 0.89 ; lo que indica que el NDVI y la temperatura del aire de alguna manera están relacionadas con la temperatura de superficie mostrando estas variables una explicación hasta del $80 \%$ de la temperatura de superficie.

Tabla 4. Coeficiente de correlación (r) y de Determinación (r2) en las relaciones entre (temperatura de superficie menos temperatura del aire, en ${ }^{\circ} \mathrm{C}$ ) y el NDVI.

\begin{tabular}{lcc}
\hline \multicolumn{1}{c}{ Fechas } & $\mathrm{r}$ & $\mathrm{r}^{2}$ \\
\hline $11 / 02 / 2008$ & 0.6709 & 0.45 \\
$15 / 04 / 2008$ & 0.8922 & 0.8 \\
$01 / 05 / 2008$ & 0.1296 & 0.02 \\
$31 / 01 / 2010$ & 0.1857 & 0.03 \\
$07 / 03 / 2011$ & 0.547 & 0.3 \\
\hline
\end{tabular}

\section{Conclusiones}

Se obtuvo un mapa de la distribución espacial de las Islas de Calor en Lima Metropolitana; se encontró la presencia de una isla de calor negativa durante la mañana y en los meses cálidos.
Se encontró en Lima Metropolitana una configuración de 15 islas de calor. Las islas de calor con los núcleos térmicos más elevados en los distritos de Ate y Lurigancho, en el distrito de Villa María del Triunfo y en el distrito de San Juan de Lurigancho. Además se observó la presencia de islas de calor en el distrito de La Molina, en los distritos de San Juan de Miraflores; Villa El Salvador y Villa María del Triunfo y en los distritos de Rímac y el Agustino.

Las islas de calor en las zonas comerciales e industriales de Lima Metropolitana estan ubicadas en las zonas industriales de los distritos de Los Olivos e Independencia, al costado de aeropuerto Jorge Chávez, en los distritos del Callao; Carmen de La Legua Reynoso; Lima y Breña, en los distritos de San Luis; Ate; El Agustino y Santa Anita y en el distrito de Surquillo, en la zona comercial del distrito de Lima y en la zona comercial e industrial de La Victoria. Asimismo se observó islas de calor en zonas con mucho asfalto en el distrito de Santiago de Surco, en el distrito de San Juan de Miraflores y en el aeropuerto Jorge Chávez.

Debido a la poca información disponible para relacionar la temperatura del aire y la temperatura de superficie no es posible afirmar la baja correlación existente entre estas variables; asimismo los coeficientes de determinación entre la temperatura de superficie y el NDVI son bajos debido a las pocas áreas verdes en el área de estudio; sin embargo al relacionar el NDVI con la diferencia de temperatura de superficie (LST) con la temperatura del aire (Ta) se encontró mejores coeficientes, así llegando hasta 0.8 el coeficiente de correlación; si la diferencia (LST - Ta) está asociada al NDVI implica que la temperatura de superficie es una variable dependiente tanto de la temperatura del aire como del NDVI.

\section{Agradecimientos}

Al Observatorio Alexander Von Humboldt de la Universidad Nacional Agraria La Molina y al Servicio Nacional de Meteorología e Hidrología del Perú (SENAMHI) por los datos meteorológicos proporcionados.

\section{Literatura citada}

Altas Ambiental de Lima. 2007. Sistema natural. Sistema humano (en línea). Lima Perú. Consultado: 30 de mayo del 2011. Disponible en http://geoserver.itc.nl/ lima/start/start.html.

Barros, PM. 2010. Modificaciones térmicas en la ciudad de Lima: análisis de la presencia de la isla de calor urbana. Tesis Ing. Ambiental. UNALM. Lima, Perú. 
CDC-UNALM. Centro de Datos para la Conservación de la Facultad de Ciencias Forestales de la UNALM. 2011. Protocolo de Ortorectificación de Imágenes Satelitales Landsat. Lima, Perú.

Chander, G. Markaham, BL y Helder, DL. 2009. Sumary of current radiometric calibration coefficients for Landsat MSS, TM, ETM+ nad EO-1 ALI sensor. Remote Sensing of Environment 113 (2009) 893-903.

Chuvieco, E. 2000. Fundamentos de Teledetección Espacial. Tercera edición. España. Reimpresión corregida 2000.

EPA (Environmental Protection Agency). Heat Island Impacts (en línea). Consultado 3 de febrero del 2012. Disponible en http:/www.epa.gov/heatisland/impacts/ index.htm.

Farina, A. 2012. Exploring the relationship between land surface temperature and vegetation abundance for urban heat island mitigation in Seville, Spain (en línea). Tesis de Master en Geographical Information Science. Lud University. LUMA GIS, tesis 15. Consultado: 10 de marzo del 2013. Disponible en http://lup.lub.lu.se/luur / download? func $=$ downloadFile \& recordOId $=3460284 \&$ fi leOId $=3460402$.

Fernandes, G. y Moreira de Souza, N. 2013. Uso de imágenes Landsat TM 5 para análise da temperatura de superficie do Distrito Federal. (1869-1876), Anais XVI Simpósio Brasileiro de Sensoriamento Remoto. SBSR; Foz de Iguaçu, PR, Brasil.

Galindo, IG 2011. Identificación y estudios de las islas urbanas de calor de las ciudades de Guadalajara y Colima, propuestas de estrategias de mitigación (en línea). Consultado el: 20 de abril del 2011. Disponible en http://www.conacyt. gob.mx/fondos/FondosSectoriales/ CONAVI/Documents/8-IgnacioGalindo.pdf.

Goldreich, Y. 1985. The estructure of the ground-level heat island in a central business district. Revista J. Climate Appl. Meteor. (24): 1237-1244.

Li, Z. Guo, X. Dixon, P y He, Y. 2008. Applicability of Land Surface Temperature (LST) estimates from AVHRR satellite image composites in northern Canada (en línea). Prairie Perspectives (11): 119 - 129. Consultado 17 de Diciembre del 2012. Disponible http://pcag.uwinnipeg. ca/Prairie-Perspectives/PP-Vol11/Li-Guo-Dixon-He.pdf.

Martínez, JF. Herrero, J y Casterad, MA. 2010. Simposio Internacional SELPER. (XIV, 2010, Guanajuato, México). Utilidad de la banda térmica de Landsat en la discriminación de terrenos yesosos. Memorias del XIV Simposio Internacional SELPER. Guanajuato, México. Sociedad de Especialistas Latinoamericanos en Percepción Remota (SELPER). 2010. 4 p.

Ministerio del Ambiente, 2014; Dirección General de Ordenamiento Territorial (DGOT). Protocolo: Ortorectificación de Imágenes Satelitales Landsat. Lima, Perú.
Pérez, C y Peña MA. Urban heat island (en línea). Consultado: 3 de febrero del 2012. http://www. urbanheatislands.com/heat-island-types.

Pulache, W y Menis, L. 2009. Cambio climático en el Perú: Impactos económicos y sociales. Climatología urbana y el cambio climático en Lima Metropolitana SENAMHI. Lima, Perú.

Quezada, JM. 1987. Estudio del Confort Climático en Lima Metropolitana y Callao. Tesis Ing. Meteorólogo UNALM. 227 p. Lima, Perú.

Roth, M.. Oke, TR y Emery, WJ. 1989. Satellitederived urban heat islands from three coastal cities and the utilization of such data in urban climatology. International Journal of Remote Sensing (10), Número 11: 1699-1720.

Sarricolea, EP y Romero, H. 2010. Análisis de los Factores Condicionantes sobre las Temperaturas de Emisión Superficial en el Área Metropolitana de Valparaíso. Arquitectura, Ciudad y Entorno 14: 79-96. Chile.

Streutker, DR. 2003. Satellite-measured growth of the urban heat island of Houston, Texas. Remote Sensing of Environment (85): 282-289.

Sun Donglian. y Kafatos Menas. 2007. Note on the NDVI-LST relationship and the use of temperaturerelated drought indices over North America. Revista GEOPHYSICAL RESEARCH LETTERS. (34). 